Meta

Journal des traducteurs

Translators' Journal

\title{
Le préconscient traducteur
}

\section{René Kaës}

Volume 40, numéro 3, septembre 1995

La traduction, qu'est-ce à dire? Phénoménologies de la traduction

URI : https://id.erudit.org/iderudit/003280ar

DOI : https://doi.org/10.7202/003280ar

Aller au sommaire du numéro

Éditeur(s)

Les Presses de l'Université de Montréal

ISSN

0026-0452 (imprimé)

1492-1421 (numérique)

Découvrir la revue

Citer cet article

Kaës, R. (1995). Le préconscient traducteur. Meta, 40(3), 478-481.

https://doi.org/10.7202/003280ar

\section{Résumé de l'article}

Freudian psychoanalysis suggests the existence of a mechanism for interpreting and signifying the reactions of others. For the author, this is a type of translation activity occurring in the preconscious, which he examines from the viewpoint of intersubjectivity, that is, as a vehicle for creativity and interpretation. His analysis is based on experiments of group interdiscursivity and of crosscullural encounters.
Ce document est protégé par la loi sur le droit d'auteur. L'utilisation des services d'Érudit (y compris la reproduction) est assujettie à sa politique d'utilisation que vous pouvez consulter en ligne.

https://apropos.erudit.org/fr/usagers/politique-dutilisation/ 


\title{
LE PRÉCONSCIENT TRADUCTEUR
}

\author{
RenE KaES \\ Universite Lumiere Lyon-2. Lyon. France
}

\begin{abstract}
Freudian psychoanalysis suggests the existence of a mechanism for interpreting and signifying the reactions of others. For the author, this is a type of translation activity occurring in the preconscious, which he examines from the viewpoint of intersubjectivity, that is. as a vehicle for creativity and interpretation. His analysis is hased on experiments of group interdiscursivity and of crosscullural encoumers.
\end{abstract}

\section{WEIN APPARAT ZU DEUTEN*}

Dans les dernières pages de Totem et tabou, Freud s'interroge sur les processus de la transmission de la vie psychique entre les générations. Il y soutient l'idée "qu'il n'y a pas de processus psychique plus ou moins important qu'une génération soit en mesure de cacher à celle qui la suit. La psychanalyse nous a montré que dans son activité spirituelle inconsciente l'homme possède un appareil qui lui permet d'interpréter (cin Apparat zu deluten) les réactions d'autres hommes, c'est-à-dire de revenir sur les déformations que ses semblables impriment a l'expression de leurs mouvements affectifs". Ainsi, la trace fait son chemin à travers les transformations qu'elle doit subir sous l'effet de la répression ou du refoulement pour passer sans être reconnue d'emblée. Cet énigmatique Apparat zu deuten dont chaque être humain serait doté n'est pas un simple appareil de décryptage, mais un appareil à signifier/interpréter les réactions et les sentiments des autres, il permet de faire retour (rï̈kgängig machen) sur le procès de signification, d'en "redresser" le sens : un tel appareil d'interprétation fabrique de nouvelles significations.

Freud suppose que cet appareil de transformation se situe dans l'activité spirituelle inconsciente de chacun (in seiner unhewußßten Geiste'stätigkeit). Peut-on considérer plutôt que cet appareil à interpréter/signifier est une forme de l'activité traductrice/interprétalive qu accomplit le préconscient? Je le pense.

Le Préconscient, comme système de l’appareil psychique, est le dispositif dans lequel s'effectuent les processus de transformation que subissent certains des contenus et des processus inconscients pour retoumer à la conscience. C'est à ce système qu'est attachée la capacité associative, traductrice et interprétative de la psyché. Si le cryptage et le décryptage peuvent qualifier l'activité du préconscient, celle-ci sera mieux spécifiée par le couple traduction/interprétation. Comme la traduction, l’interprétation est en effet une production «active», au sens recréatif que Derrida donne à ce qualificatif, pour signifier que la traduction n est pas un simple transcodage. Et, comme la traduction, l'interprétation est une activité qui s'inscrit au coeur même du lien intersubjectif. C'est sur ces dimensions que je voudrais attirer l'attention.

\section{LE PRÉCONSCIENT COMME TRADUCTION/INTERPRÉTATION}

Le préconscient et son activité doivent retenir notre intérêt pour plusieurs raisons. La première est que cette instance et celte fonction de l'appareil psychique sont encore aujourd'hui mal connues. Introduire la dimension de l'intersubjectivité pourrait ouvrir une voie de connaissance plus précise si nous parvenions à reprendre la théorie du préconscient non seulement avec la seconde théorie de l'appareil psychique. mais au-delà. c'est-à-dire dans les termes d'une métapsychologie de l'intersubjectivité.

M'fd. XL. 3. 1995 
Un second foyer d'intéret est celui-ci : je suis frappe par le fait que les souffrances intenses de la vie psychique font toujours apparaître soit des défaillances dans l'activité du preconscient, soit la non-constitution de cette instance. Ces pathologies du preconscient ne peuvent étre comprises et traitées que dans la mesure ou le travail du préconscient d'un autre (le psychanalyste, le psychothérapeute) ou de plus-d'un-autre, reconstitue les conditions d'une relance de l'activité de symbolisation jusqu'alors defaillante ou inadvenue. On peut qualifier le travail du préconscient de l'autre ou de plusd'un-autre essentiellement en termes de leur activité créatrice. traductrice et interprétative. Une telle activité appelle la capacité de rêverie décrite par Winnicott : elle se produit par l'invention de paroles adressées à un autre, probablement d'abord à cet autre en soi-même, témoin de l'altérité interne, double étrange familièrement inconnu.

\section{LE PRECONSCIENT COMME SYSTEME DE TRANSFORMATION}

La formation du Préconscient suppose le refoulement secondaire, la constitution d'une capacité de transformation et de rétention des contenus inconscients. Le Préconscient est le lieu des inscriptions de mots, le lieu des montages psychiques qui tiennent leurs origines des toutes premières expériences de l'infans dans son rapport à la parole et au langage. Cette expérience est sous le sceau du porte-parole que la mère a été pour lui, P. Aulagnier l'a fortement montré ; s'y constituent conjointement certaines conditions, intersubjectives, du refoulement et du retour de refoulé.

"Les mots qui vont surgir savent de nous ce que nous ignorons d'eux", écrit René Char. Chaque parole qui se forme est comme une naissance a la relation d'inconnu. Dans le préconscient, les mots savent et ne savent pas ce qu'ils vont servir : tout aussi bien le refoulement que le frayage des voies du retour du refoulé. Le préconscient est ouvert dans ses deux directions comme un commutateur psychique qui porte trace de ses premières expériences constituantes.

L'activité du préconscient suppose comme condition de sa possibilité un premier travail de symbolisation. À partir de cette première donnée, le sujet fait l'expérience que la réalité interne et externe est transformable en sens, qu'elle a acquis un certain degré de familiarité et qu'elle inspire suffisamment confiance.

L'activité du Préconscient de l'autre est particulièrement sollicitée dans les expériences de crise, chaque fois que le préconscient du sujet est défaillant à maintenir les liens de l'association des représentations de choses et des représentations de mots avec les affects correspondants.

La question prend tout son relief lorsque l'on est confronté à des cas où le refoulement n'a pas pu s'effectuer du fait de la pathologique d'un parent psychotiquel' : les contenus non refoulés apparaissent dans le délire d'un enfant ou dans des formations clivées qui demeurent en souffrance de symbolisation. Dans de tels cas, la pensée délirante de l'enfant se substitue à ce qui a été empêché dans l'appareil à penser des parents : l'interprétation de la réalité psychique s'effectue sans les transformations qu impose le préconscient pour traduire l'inconscient.

\section{PRECONSCIENT ET INTERSUBJECTIVITÉ}

Préconscient, activité parlante et intersubjectivité sont liés d'une manière fondamentale. La formation et l'activité du Préconscient sont profondément tributaires de l'interjeu entre le sujet et l'autre: elles dépendent pour une part du Préconscient de l'autre, de sa capacité de rêverie, de contention et de transformation. La première fonction de porte-parole accomplie par la mère, celle où elle accompagne par la parole les expériences de plaisir et de déplaisir de son enfant, est la condition et le modèle de la 
formation du Préconscient à partir de l'activité de l'Autre. Le Préconscient de la mère constitue une partie de l'appareil à signifier/interpréter de l'enfant ; appareil à traduire et à transmettre les couches successives de discours qui, tel un palimpseste, se sont inscrites en lui et pour lui. Ainsi, tout comme la fonction refoulante, l'élaboration psychique préconsciente s'effectue dans le jeu intersubjectif, qu'elle contribue à soutenir. Elle définit le lieu intersubjectif de la métaphore, lieu qui s'inscrit dans l'espace du groupe primaire dans les paroles échangées autour du berceau, ou dans les bras du parent. C'est le lieu même de l'expérience transitionnelle.

\section{TRADUCTION ET INTERSUBJECTIVITÉ : L'EXPÉRIENCE DU GROUPE}

Les travaux contemporains sur les processus associatifs dans les groupes. conduits selon la méthodologie psychanalytique, ouvrent très précisément cette perspective.

Dans mon dernier ouvrage, La parole et le lien, j'ai essayé de qualifier le travail psychique qui s'effectue par le moyen du groupe à travers les formes et les modalités des transferts et des processus associatifs. J'ai été amené à définir ce type de travail psychique comme le travail de l'intersubjectivité : l'idée est qu'un autre (ou un ensemble d'autres) peut effectuer pour un sujet, dans certaines conditions, un travail de liaison et de transformation qui lui est momentanément inaccessible ou lui a été jusqu'alors impossible. Le processus associatif dans le jeu de l'interdiscursivité groupale rétablit l'activité du préconscient là où des signifiants se sont trouvés en défaut de représentation de mot.

Un exemple montre comment un membre d'un groupe fait surgir dans le processus associatif quelque chose qui lui apparaît comme énigmatique. Il se met alors en position d'attendre et d'entendre dans le développement des associations des autres membres du groupe une voie d'accès à son énigme. Les autres développent leurs propres associations tout en demeurant en association avec l'énigme de cet homme qui, à l'écoute de ce qu'ils disent, trouve dans leurs paroles le signifiant qui lui a fait défaut. La parole des autres fraie la voie au retour du refoulé. Ceci explique que. dans les groupes, certains demeurent silencieux et continuent à associer sans que la parole émerge. La question est d'essayer de comprendre le statut de la parole dans le processus associatif en groupe. J'ai proposé l'exemple de quelqu'un à qui une autre personne demande d'être son "porte-parole» et qui découvre soudain que la parole qu'il profère au nom d'un autre le concerne au plus vif de sa propre histoire : il s'éprouve lui-même parlé par la parole qu'il transporte pour un autre. Quiconque est porte-parole parle à la place d'un autre, pour un autre, mais aussi pour l'autre qui est en lui et qui trouve dans cette parole transportée un signifiant qui ne lui était pas disponible?

\section{LA LANGUE ÉTRANGËRE ET LE PRÉCONSCIENT}

Je voudrais souligner l'importance du préconscient dans la mise en représentation de mot lors de la rencontre interculturelle. Pour me faire comprendre, je vais évoquer une expérience que j'ai faite avec douze collègues européens lorsque nous avons fondé il y a quelques années l'Asscciation européenne d'analyse transculturelle de groupe. Notre projet était d’étudier par la méthode psychanalytique de groupe ce qui est en jeu du point de vue de la réalité psychique dans les rencontres entre des sujets issus de cultures différentes. Nous nous sommes réunis pendant trois ans avant de mettre en place le premier séminaire approprié à notre objectif (Maastricht 1985), chacun parlant ou comprenant plus ou moins aisément deux ou trois langues, mais très rarement toutes celles dont usaient chacun d'entre nous. Nous avions convenu qu'il $n$ 'y aurait ni langue officielle ni interprète : nous expérimentions ainsi comment se faisait le choix des langues le plus souvent usitées et, surtout, ce qui se passait en nous et entre nous lorsque nous 
n'avions pas accès à une langue. C'était là une expérience particulièrement intéressante pour des psychanalystes confrontés à déchiffrer et interpréter les messages de l'inconscient. Lors de ces réunions, il arrivait régulièrement que nous éprouvions à tour de rôle. dans des formes et avec des intensités diverses, de véritables symptômes psychosomatiques : maux de tête, petits troubles digestifs, insomnies, etc. J'étais frappé par l'expérience de violence qui accompagnait ces moments angoissants où nous ne comprenions pas, parmi d'autres qui comprenaient. Je n'étais pas satisfait par une explication en termes d'exclusion, tel l'enfant devant la langue énigmatique des parents. Je pense qu'il s'agissait plus fondamentalement d'une mise en échec momentanée de l'activité du préconscient, qui ne disposait plus des préformations linguistiques et figuratives pour acheminer les representations inconscientes et les charges d'affect qui leur sont liées vers "les mots pour le dire". J'apprenais avec mes collègues ce que les participants de nos séminaires auraient à eprouver et à symboliser : la violence psychique qui se trouve mobilisée lorsque les ressources et l'activité du préconscient sont mises en défaut, et elles le sont dans toute rencontre avec l'autre, étrange étranger. Je notai aussi combien est plus directe l'expression des affects, et moins secondarisées les représentations dont la parole rend compte lorsque nous utilisons une langue qui ne nous est pas familière.

\section{TRADUIRE L AUTRE OU LA «CULTURE DU PRECONSCIENT *}

Je trouve dans la préface écrite par Yves Bonnefoy pour sa traduction des sonnets de Shakespeare une pensée grâce à laquelle je peux penser ce que j'essaie de dire :

Le matériau du traducteur, c'est moins le "sens" qu'a le texte - un sens qui est d'ailleurs bien complexe, et se trouve ou se perd a divers niveaux - que son experience propre de celui-ci : laquelle, s'il est lui-même, et comme il se doit, un esprit inventif au monde, ne peut que prendre la forme d'une question qu'il se pose, sur la valeur, sur le bien-fondé de l'original, avec sinon son approbation, de sa part, du moins une comprehension assez ouverte. assez. sympathique pour qu'il puisse lui prêter sa voix un instant, dans ce jeu d'hypothèses qui constitue notre devenir. Si cette sone d'accord n 'etait pas possible, mieux vaudrait que le traducteur renonce à la traduction, car on ne traduit bien que son proche.

Pour traduire/interpréter l'autre, il faut assurément connaître plusieurs langues, mais il faut surtout soutenir ce rapport de proximité et d'écart avec l'autre, perdu et recré, qu'exige une identification vivante. Il nous faut développer cette culture du préconscient qui est tout à la fois culture de l'identification et capacité de traduction simultanée de ce rapport de soi avec l'autre et avec l'autre en soi. Cette culture exige que nous fassions l'expérience de ce qu'a d'effrayant notre propre étrangeté psychique, mais aussi de familier l'espace psychique de l'autre. La fonction traductrice/interprétative du preconscient se fonde sur le maintien de cet écart transitionnel entre une tautologie qui répèterait le même et un insensé qui ne dirait que l'inconnu inaccessible.

Notes

1. Sur cette question, $c f$. les travaux de $M$. Enriquez sur le edelire en heritage* et l'incidence du délire des parents sur la memoire des descendants, in R. Kals. H. Faimberg et coll.. 1993. Transmission de la vie psychique entre générations, Paris, Dunod.

2. René Kaés, 1994. La parole et le lien. Les processus associatifs dans les groupes. Paris. Dunod. 